\title{
The Random Walk Model Based on Bipartite Network
}

\author{
Man- Dun Zhang ${ }^{1,2}$, Shun-Shun Chang ${ }^{1,2}$, Jia-Wei Zhao ${ }^{1,2}$ and Jian-Hong Ma ${ }^{1,2}$ \\ ${ }^{1}$ School of computer science and engineering, Hebei University of Technology \\ ${ }^{2}$ Hebei province key laboratory of big data calculation, China \\ Corresponding Author: Jianhong Ma, majianhong@scse.hebut.edu.cn \\ E-mail: ${ }^{a}$ zhangmandun@scse.hebut.edu.cn, ${ }^{b} 846176284 @ q q . c o m,{ }^{c}$ zhaoJwvsMike@outlook.com
}

\begin{abstract}
With the continuing development of the electronic commerce and growth of network information, there is a growing possibility for citizens to be confused by the information. Though the traditional technology of information retrieval have the ability to relieve the overload of information in some extent, it can not offer a targeted personality service based on user's interests and activities. In this context, the recommendation algorithm arose. In this paper, on the basis of conventional recommendation, we studied the scheme of random walk based on bipartite network and the application of it. We put forward a similarity measurement based on implicit feedback. In this method, a uneven character vector is imported(the weight of item in the system). We put forward a improved random walk pattern which make use of partial or incomplete neighbor information to create recommendation information. In the end, there is an experiment in the real data set, the recommendation accuracy and practicality are improved. We promise the reality of the result of the experiment
\end{abstract}

\section{Introduction}

Unlike the technology of information retrieval, the information filtering technology, in some extent, can alleviate the information overload, and the personalized recommendation technology is one of the most successful application of it. The core of a recommendation system is the recommendation algorithm. These algorithms or models have many functions: simulating users' behavior, predicting users' interests and recommending items that users may buy or interests in. In the existing recommendation algorithms, the collaborative filtering is the most widely used and effective algorithm. It can predict users' preference of a new product by their neighbors' historical behavior. Under normal circumstances, the collaborate filtering consists of three parts: 1.A measurement method calculating the similarity between user-user and product-product;2.Intergrating the similarity measurement method to create the recommended list.3.Getting the best performance patterns by adjusting the data trace.

Collaborative filtering is generally applied in recreational products like films and music. Yet when it comes to a system consists of products that can be bought repetitively, the collaborative filtering is not widely used. In addition, many existing recommendation algorithms are based on matrix of products' score grated by users. Generally speaking, these scores are classified obviously, and they represent the users' interest from lowest to highest level clearly by discrete values. However, the items generally transfer implicit motion data, so the expression of specifically fixed values is unreasonable..

\section{Correlation Technique}

\subsection{Similarity}

The key to recommendation algorithm is similarity technique. For example, the literature[1] proposed a similarity measure method based on information entropy. Literature[2] provided a method of clustering to find similar users in order to produce recommendations. They both achieved some effect. In general, in a recommendation system, there is a set consists of user $u$ and products $i$, and some scores of products purchased by user $u$. The aim of recommendation system is to predict some other products' scores grated by users, and recommend the product set i users u might like. In the following part, we take cosine similarity as an example to introduce similarity algorithm. Cosine similar degree, also known as cosine similarity, is a assessment of 
similarity by calculation the angle between two cosine vectors.

$$
\operatorname{sim}(i, j)=\frac{R_{\bullet, j} \cdot R_{\bullet j}}{\left|R_{\bullet, i}\right|\left|R_{\bullet j}\right|}
$$

In this formula, the vector R represents the item v's score matrix grated by user $u$. And is the expression of the line i score vector of $\mathrm{R}$.

\subsection{The model of random walk}

The model of random walk originate from google'spagerank algorithm. This algorithm is firstly used to calculate the importance of web pages. The importance of web pages expressed in formula is as follows:

$$
R^{n}=d \cdot P \cdot R^{n-1}+(1-d) \cdot U
$$

\section{The random walk model based on Bipartite network}

In order to solve existing problems by using the method presented previously, we put forward a improved random walk model. In the algorithm this paper proposed, similarity is defined as the transition probability of bipartite network. The relationship between the items is simulated by the random walk model.

\subsection{The first-order transition probability}

Shopping data can be described as a node of a bipartite network: customer and product. In the network, every edge represents customer's purchase frequency of corresponding product. The user set can be described as $\mathrm{U}=$, and the item set is described as $\mathrm{V}=$. The customer's purchase frequency of products can be expressed as $\mathrm{F}=\{\mathrm{f}(1,1), \mathrm{f}(1,2), \ldots \ldots \mathrm{f}(\mathrm{u}, \mathrm{v})\}$.

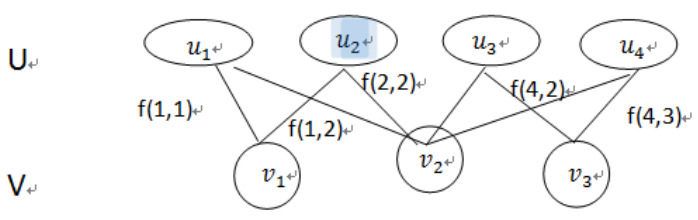

Figure 1. User-Item Bipartite Network

From the point of view of random walk, the conditional probability of under , $\mathrm{P}(\mid)$, can be expressed as the probability of random walk from to, and the formula of it is

$$
P\left(v_{j} \mid v_{i}\right)=\sum_{k=1}^{|u|} P\left(v_{j} \mid u_{k}\right) P\left(u_{k} \mid v_{i}\right)
$$

In this formula, the represents the probability of random walk from the product node to the user node . denotes the probability of random walk from the customer node to product node. $\mathrm{P}(\mid)$ refers to the sum of all the marginal probability. Generally speaking, $\mathrm{P}(\mid)$ is called the popularity of products among users who purchased.

Literature[1] regards $\mathrm{P}(\mid)$ as the first-order similarity between users and products. The definition of similarity based on the condition that users have bought both of the products. But the truth is two items can be similar even if they are not bought together. It is obvious when the data is sparse. And we will discuss it in the following research. In this paper, is estimated as the quotient between the time buy and purchase history. The formula is:

$$
P\left(v_{j} \mid u_{k}\right)=\frac{f\left(u_{k}, v_{j}\right)}{\left(\sum f\left(u_{k^{*}}\right)\right)^{\alpha_{1}}}(4)
$$

In the same way:

$$
P\left(u_{k} \mid v_{i}\right)=\frac{f\left(u_{k}, v_{i}\right)}{\left(\Sigma f\left(k, v_{i}\right)\right)^{\alpha_{2}}}
$$

$\in[0,1]$ are used to adjust the effects brought by data sparse and data redundancy

\section{2 the random walk based on bipartite network}

Now we apply the first-order similarity to the whole bipartite network, and we can infer that:

$$
\mathrm{P}^{*}=\sum_{\mathrm{n}=1}^{\infty} \frac{(\mathrm{dP})^{\mathrm{n}}}{\left|(\mathrm{dp})^{\mathrm{n}}\right|}
$$

In formula(6), $\mathrm{P}$ is calculated by the formula(4), $\mathrm{d} \in$ $[0,1]$ is called damping coefficient. Obviously we can conclude that: when $\mathrm{n}$ tends to infinity, converges to .

$\mathrm{P}$ is directly got by the user's purchase behavior. In order to get the recommendation set $\mathrm{R}$, we improved the 
model by adjusting and biasing. A heterogeneous personality vector called $U$ is imported in every random walk model iteration. The formula is:

$$
R=d \cdot P \cdot R+(1-d) \cdot U
$$

In the formula(7), $\mathrm{R}$ represents the set of vital parameters in the recommendation order. When the item number is $i,=1 / \mathrm{m}, \mathrm{m}$ is called the purchase frequency of the users in figure 1 . In other conditions,

Then we will do further calculation and derivation of formula $(8)$. To reduce the response time, we import a fast approaching method:

Then we call the recommended item's major parameter. $\mathrm{m}$ denotes the product's purchase frequency in the purchase history. Compared with $\widehat{R}, \mathrm{R}$ improves the accuracy of the recommendation list, and we call this model the Random Walk model based on Bipartite Network(BNRW)

$$
\begin{aligned}
& \hat{\mathrm{R}}=\sum R_{i} \\
R_{i} & =d \cdot P \cdot R_{i}+(1-d) \cdot U_{i} \\
R & =\frac{1-d}{1-d \cdot p} U \\
& =\frac{1-d}{1-d \cdot p} \sum \frac{U_{i}}{m} \\
& =\frac{1}{m} \sum R_{i}
\end{aligned}
$$

\section{Experiment}

\subsection{Judgement Criteria}

With the development of the recommendation system, there are many judgement criteria of recommendation precision. There are three other judgements in the field of information retrieval and personality recommendation: precision, recall and F-measure. In this paper, we use the method of literature(3) called Weighted Hit Rates as the judgement. The formula is:

$$
\mathrm{wHR}=\frac{\sum_{\mathrm{i}}\left(1-p\left(x_{i}\right)\right) * H I T\left(x_{i}\right)}{\sum_{\mathrm{i}}\left(1-p\left(x_{i}\right)\right)}
$$

represents item i, $\mathrm{p}()$ denotes prior probability. When it is hit, $\operatorname{HIT}()=1$ and on the contrary $\operatorname{HIT}()=0$.

\subsection{Data Set}

In this paper, all the data comes from the purchase history of a market in China, and it can be described as follows:

Table 1 data set description

\begin{tabular}{llll}
\hline & customer & product & behavior \\
$\begin{array}{l}\text { training } \\
\text { set }\end{array}$ & 88000 & 846321 & 78625165 \\
testing set & 12000 & 45789 & 236548 \\
\hline
\end{tabular}

\subsection{Result}

We regard every customer as a single carrier, so the purchase frequency is a vital property

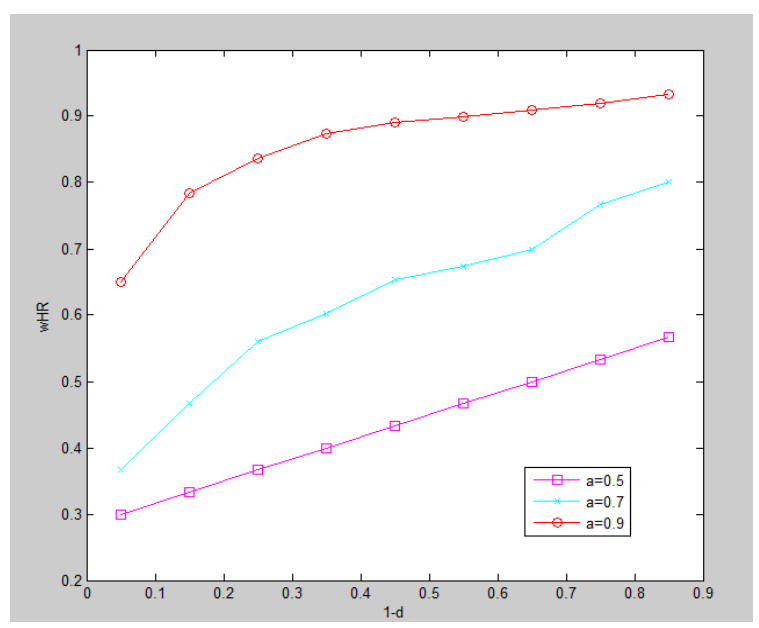

Figure 2 parameter analysis

Now we find appropriate by continuing fitting data.

In figure 2 , the $\mathrm{x}$ coordinate denotes $1-\mathrm{d}$, and $\mathrm{y}$ ordinate represents hit rate(wHR). The three lines represents the results of respectively. We can infer from the figure 2 that when, the result of the experiment reaches a peak. Then there's a compare between the conventional algorithms of cosine, content-base CF and project-based. And the result is in the following figure:

Table 2 compare of data set between algorithms of BNRW

\begin{tabular}{lllll}
\hline & Precision & Recall & Fmeasure & wHR \\
BNRW & 0.6573 & 0.5864 & 0.5168 & 0.7653 \\
CBCF & 0.5372 & 0.5126 & 0.5053 & 0.6010 \\
PBCF & 0.5139 & 0.4986 & 0.5042 & 0.5886 \\
Cosine & 0.4568 & 0.4856 & 0.4986 & 0.6046 \\
\hline
\end{tabular}

In the figure 3 below, there is the wHr distribution situation of part of users: 


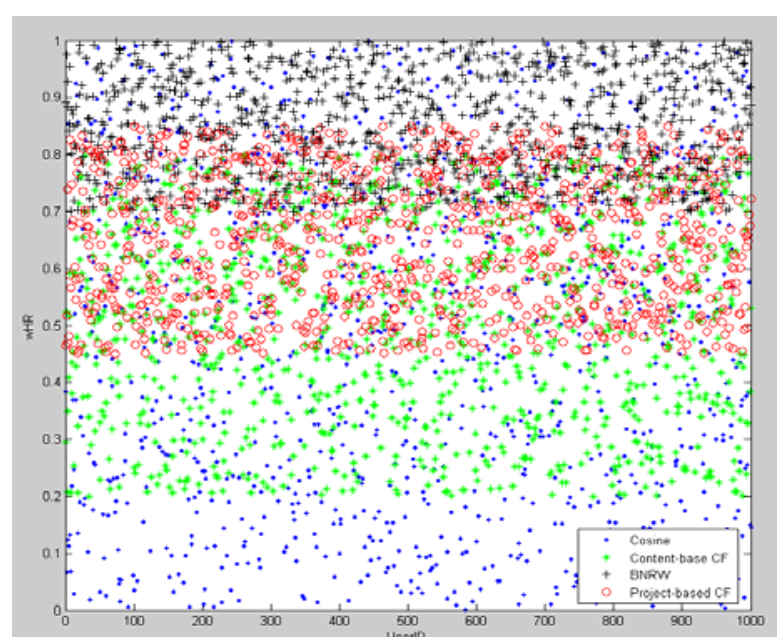

Figure $3 \mathrm{Whr}$ distribution figure of part of users

\section{Conclusions}

This paper put forward an improved random walk model based on bipartite network. The item's weight in system is imported, with the parameter, we can find the area close to user and create the recommendation. The experiment traced the data in a real data set, compared the difference between other two algorithms, the hit race improved obviously. However, the recommendation algorithm is always easy to learn and hard to master, and our future goal is to make recommendation results perform better in diversity and precision.

\section{References}

1. XiaPeiyong, Ding Xiangqian, Research on Collaborative Filtering Algorithm of Personalized Recommendation Technology[d], Ocean University of China, 2011.

2. Jin Di, Yang Bo, Liu Jie, Liu Dayou, He Dongxiao. Ant Colony Optimization Based on Random Walk for Community Detection in Complex Networks[J]. Journal of Software. 2012

3. Malcolm Benjamin Dias, Jan Jarman, Grocery shopping recommendations based on basket-sensitive random walk,2016.4.

4. He Jianjun, Li Renfa. Modified nodes ranking methods using random walk model. Computer Engineering and Applications,2011,47(12):87-89

5. Guo Lei, Ma jun, Chen Zhumin, Jiang Haoran. Incorporating Item Relations for Social Recommendation[J]. 2014, 37(1):219-226.

6. Ma H, King I,L Yum, Learning to recommend with social trust ensemble//Proceeding of the 32nd Internationnal ACM SIGIR Conference on Reserch
and
Develoment in
Information

Retrieval.Boston,USA,2009:203-210

7. Xiang Liang. Recommendation in Practice. Beijing: Postd \& Telecom Press,2012(in Chinese).

8. Gleiser PM, Danon L. Community structure in jazz. Advances in Complex Systems, 2003,6(4):565 573. [doi: 10.1142/ S0219525 903001067].

9. Zachary WW. An information flow model for conflict and fission in small groups. Journal of Anthropological Research, 1977, 33(4):452 473.

10. Fortunato S, Barthélemy M. Resolution limit in community detection. Proceedings of National Academy of Science, 2007,104(1): 36 41. [doi: 10.1073/pnas.0605965104]

11. Chen Dengke. Hybrid Lantent Vector Model-and Item-based Collaborative Filtering Recommendation[D], Zhejiang University.2010

12. Chen T,TangL, Combining factorization model and additive forest for collaborative followee recommendation[J].KDD CUP,2012 hed) 